\title{
Prise en compte du mouvement dans les systèmes de communication sans-fil
}

Arnaud Troël, Frédéric Weis, Michel Banâtre

\section{$\mathbf{N}^{\circ} \mathbf{4 7 2 0}$}

Fevrier 2003

THÈME 1 



\title{
RINRIA
}

\section{Prise en compte du mouvement dans les systèmes de communication sans-fil}

\author{
Arnaud Troël, Frédéric Weis, Michel Banâtre* \\ Thème 1 - Réseaux et systèmes \\ Projet ACES
}

Rapport de recherche $\mathrm{n}^{\circ} 4720$ - Fevrier 2003 - 46 pages

Résumé : Fruits des progrès de l'informatique embarquée et des technologies de communication sans-fil, des terminaux d'un nouveau type sont récemment apparus. Disposant d'une capacité de calcul non négligeable, ils peuvent de plus échanger des informations directement avec leurs pairs ou bien par le biais d'un réseau relais.

La portée de communication dont ils disposent étant limitée, le mouvement de ces terminaux joue un rôle immédiat dans le fonctionnement de leurs échanges. La prise en compte de cette mobilité apparaît alors comme une perspective intéressante à développer lors de l'élaboration de systèmes mettant en œuvre ces terminaux.

L'objectif de ce document est d'examiner et de classifier les différentes techniques employées afin de permettre aux systèmes informatiques d'effectuer cette prise en compte. Nous verrons qu'elle dépasse le cadre des systèmes adaptatifs.

Mots-clé : mobilité, communications sans-fil, ubiquité numérique

(Abstract: pto)

* \{Arnaud.Troel, Frederic.Weis, Michel.Banatre $\} @$ irisa.fr 


\title{
Taking motion into account in wireless systems
}

\begin{abstract}
Recent advances in the fields of embedded computing and wireless communications technologies have allowed to a new type of terminals to emerge. Taking advantage of fit capacities in terms of computing, they also can exchange data both directly or through a specific network.

Because their communication range is limited, the motion of these terminals has an immediate impact on how their exchanges work. Taking into account this motion seems to be an interesting perspective for systems employing such terminals.

The objective of this document is to investigate and classify the different ways in which this motion is taken into account by computing systems. We will see that this goes beyond adaptative systems.
\end{abstract}

Key-words: motion, wireless communications, ubiquitous computing 


\section{Table des matières}

1 Introduction $\quad 5$

2 Mobilité et systèmes de communication sans fil 7

2.1 Mobilité physique : définition et détection . . . . . . . . . . 7

2.1 .1 Définition . . . . . . . . . . . . . 7

2.1.2 Détection de la mobilité . . . . . . . . . . 8

2.2 Systèmes mobiles de communication sans fil . . . . . . . . . . . . . 13

2.2 .1 Les réseaux cellulaires . . . . . . . . . . . 13

2.2 .2 Les réseaux ad hoc . . . . . . . . . . . . 15

2.2.3 Les réseaux de personnes . . . . . . . . . . . 17

2.3 Synthèse . . . . . . . . . . . . . . . . . . . 18

3 Application aux approches adaptatives $\quad \mathbf{2 0}$

3.1 Présentation de l'approche adaptative . . . . . . . . . . . . . 20

3.2 Prise en compte spatiale de la mobilité . . . . . . . . . . . . . 22

3.2.1 Réservation de ressources dans les réseaux cellulaires . . . . 22

3.2.2 Cas du protocole de routage ad hoc LAR . . . . . . . . . . 24

3.2.3 Cas du protocole de routage ad hoc DREAM . . . . . . . . 25

3.3 Prise en compte temporelle de la mobilité . . . . . . . . . . . . . . 27

3.3.1 Anticipation du handover dans les réseaux cellulaires . . . . 27

3.3.2 Durée de voisinage dans les réseaux ad hoc . . . . . . . 28

3.3.3 Messages de contrôle dans les réseaux ad hoc . . . . . . . . 30

3.3.4 Durée de voisinage dans les réseaux de personnes . . . . . . 31

3.4 Synthèse . . . . . . . . . . . . . . . . . . . 33

4 Application aux approches ubiquitistes $\quad 34$

4.1 Présentation de l'approche ubiquitiste . . . . . . . . . . . . . . . . 34

4.2 Adressage dynamique par le mouvement . . . . . . . . . . . . 35

4.2 .1 Cooltown . . . . . . . . . . . . . 36

4.2 .2 Spread ...................... 36

4.3 Mouvement comme médium de communication . . . . . . . . . . 37

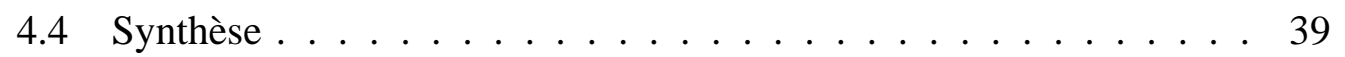

5 Conclusion $\quad 40$

RR $n^{\circ} 4720$ 


\section{Table des figures}

1 Accès par bornes à une infrastructure centralisée . . . . . . . . . . . . 14

2 Réseau cellulaire . . . . . . . . . . . . . . . . . . 14

3 Réseau ad hoc . . . . . . . . . . . . . . . . . 15

4 Réseau de personnes . . . . . . . . . . . . . . . . 18

5 LAR: request zone et expected zone . . . . . . . . . . . 25

6 DREAM: calcul de l'angle de routage . . . . . . . . . . . 26

7 calcul du temps d'expiration du lien dans les réseaux ad hoc . . . . 29

8 Prédiction de la durée de voisinage par régression . . . . . . . . . . 32

9 Système d'informations spatial . . . . . . . . . . . . . . . . . 37

10 Les différents échanges dans un réseau Pollen . . . . . . . . . . . . . . . 38

11 Taxonomie de la prise en compte du mouvement . . . . . . . . . . . 41 


\section{Introduction}

Les progrès technologiques conjoints dans des domaines tels que l'informatique embarquée, la communication sans fil et la consommation énergétique ont permis l'émergence de terminaux informatiques d'un nouveau type . D'une part, grâce au faible encombrement qu'ils engendrent et la grande capacité d'autonomie dont ils disposent, ces terminaux sont aisément transportables par des piétons, des véhicules automobiles ou encore des robots. D'autre part, la présence d'une interface de communication sans fil leur apporte une autre spécificité essentielle, celle de pouvoir échanger de l'information avec leurs pairs directement ou par le biais d'un réseau cellulaire. Les assistants personnels et les téléphones portables sont aujourd'hui des exemples très répandus de ce type de terminaux.

Les interfaces de communication qui équipent ces terminaux ont une portée limitée. Sans recourir à infrastructure supplémentaire pour relayer ses messages, un terminal ne peut communiquer qu'avec ses voisins directs, c'est-à-dire les entités se tenant à sa portée. Or, étant à la fois portables et autonomes, ces terminaux subissent les mouvements de leur utilisateur. De ce fait, l'ensemble des entités voisines d'un terminal varie en fonction de leurs déplacements. Considérons, en guise d'illustration, le domaine bien connu de la téléphonie mobile. L'usager dispose d'un terminal à l'aide duquel il doit pouvoir joindre un autre usager, par le biais du système de téléphonie. La portée d'un téléphone portable étant limitée, cet accès est réalisé grâce à d'un réseau d'antennes réparti dans la zone couverte par le système. Le déplacement de l'usager peut, dès lors, induire des changements dans l'ensemble des entités voisines (les antennes à portée). L'accès au réseau doit donc être effectué via une antenne dont le choix dépend de la localisation du terminal. Un mouvement de sa part peut nécessiter une modification de point d'accès.

Ce constat de l'étroite relation existant entre mobilité et communication laisse entrevoir le rôle singulier que la mobilité physique joue inévitablement dans le fonctionnement des systèmes composés de ce genre de terminal. La prise en compte de leur mobilité physique apparaît alors comme une perspective intéressante dans l'élaboration de tels systèmes.

La prise en compte de la mobilité des terminaux par un système peut, par exemple, permettre à ce dernier de s'y adapter afin d'améliorer les services qu'il offre. Cela renvoie à la problématique de l'adaptation du système informatique vis-à-vis de la mobilité. Ces systèmes adaptatifs ont été étudiés de manière no- 
table $[14,21,9]$. Cette prise en compte de la mobilité est également nécessaire dans d'autres domaines, tels que celui de l'ubiquité numérique. Introduite par Weiser [47], son objectif principal est de rendre le recours par l'utilisateur aux moyens informatiques plus intuitif et transparent. Pour cela, un ensemble de terminaux mobiles et communiquants est généralement mis en œuvre [45, 22]. La prise en compte de leur mobilité est là encore très souvent nécessaire au fonctionnement du système considéré.

Ce document propose d'examiner et de classifier les différentes techniques employées afin de permettre aux systèmes informatiques de prendre en compte cette mobilité. Nous l'envisageons de manière générale, quitte à laisser de côté les problèmes techniques relatifs à l'utilisation d'une technologie ou d'un réseau particulier. S'ils sont d'un intérêt indéniable, ils ont déjà été traités pour la plupart, ou apportent peu à l'étude proprement dite. Nous verrons ainsi que la prise en compte de la mobilité physique dans les systèmes de communication sans fil dépasse le simple cadre adaptatif.

Dans un premier temps, nous présentons les domaines auxquels ce document fait référence. Nous définissons ce que nous entendons par le terme mobilité et évoquons brièvement les différentes techniques permettant de la détecter au niveau informatique. Nous rappelons ensuite les principales catégories de réseaux de communication sans fil dans lesquels les terminaux mobiles évoluent principalement. Dans le chapitre suivant, nous examinons les différentes méthodes de prise en compte de la mobilité dans une approche adaptative traditionnelle et montrons les apports de ces techniques. Nous réservons au quatrième chapitre l'approche nouvelle par l'ubiquité numérique de la prise en compte de la mobilité, dans laquelle le mouvement joue un rôle essentiel dans les systèmes informatiques considérés. Enfin, nous concluons en proposant une taxonomie originale des différentes méthodes de prise en compte de la mobilité dans les systèmes de communication sans fil. 


\section{Mobilité et systèmes de communication sans fil}

Ce chapitre permet de définir et de rappeler diverses notions dont il sera fait référence dans la suite de document. La première partie est consacrée à ce nous avons évoqué précédemment sous les termes de mobilité physique. Afin de lever toute ambiguïté sur cette notion, nous en donnons tout d'abord la définition. Nous évoquons ensuite les diverses méthodes permettant de la détecter. Le thème de la seconde partie est, quant à lui, celui des systèmes de communications sans-fil. Nous y présentons les différentes formes sous lesquelles ces systèmes se déclinent et montrons plus précisément en quoi la mobilité des terminaux les composant peut y jouer un rôle non négligeable.

\subsection{Mobilité physique : défi nition et détection}

Dans cette partie, nous nous intéressons plus précisément à la notion de mobilité physique. Comme nous l'avons souligné dans le chapitre précédent, mobilité et communication sont fortement corrélées dans les systèmes sans fil. Afin d'offrir un service de bonne qualité, il peut s'avérer judicieux de prendre en compte et d'exploiter activement la mobilité des terminaux. Pour cela, il est nécessaire d'être en mesure de percevoir cette mobilité au niveau informatique. Après avoir tout d'abord défini précisément ce que nous entendons par mobilité physique, nous évoquons les différentes technologies disponibles permettant de la détecter.

\subsubsection{Définition}

Le terme de mobilité est très utilisé dans le domaine informatique. Généralement, le concept de mobilité désigne une modification d'état, qu'il soit concret ou abstrait. Dans le domaine des agents mobiles, la mobilité est un phénomène qui désigne le saut d'un processus informatique (l'agent) d'une ressource informatique à une autre (par exemple, un navigateur Web qui passe d'un URI à un autre). La mobilité est considérée exclusivement sous l'angle informatique. Les mécanismes de roaming renvoient, eux, au domaine des réseaux. Ils permettent à une machine d'être mobile, c'est-à-dire de changer de réseau de manière transparente du point de vue de l'adressage. La mobilité se manifeste, vis-à-vis du réseau, comme une modification du système d'adressage. 
Dans ce document, la notion de mobilité à laquelle nous faisons référence est la mobilité au sens commun, telle que nous la percevons tous les jours. Nous emploierons les termes de mobilité, mobilité physique ou encore plus simplement mouvement pour désigner cette notion.

Les terminaux envisagés sont des terminaux de taille réduite, facilement portables par un piéton ou par un véhicule. Dès lors, ces calculateurs, ou entités communicantes, subissent directement les mouvements de l'entité physique qui les transporte. Cela leur confère de facto une mobilité physique. Par la suite, nous assimilerons entité physique et entité communicante en tant qu'entité, terminal, ou nøud. De ce fait, la mobilité du terminal est considérée comme une mobilité a priori, c'est-à-dire qu'elle lui est intrinsèque. C'est précisément la prise en compte de cette mobilité que nous proposons d'étudier.

Il est important de noter que la mobilité ne se limite pas à la simple localisation. Le mouvement est une notion cinématique, c'est-à-dire qu'il repose sur une relation entre le temps et l'espace. Une position ne suffit donc pas à le décrire. La description minimum d'un mouvement est un historique de localisations datées.

\subsubsection{Détection de la mobilité}

Le besoin de la prise en compte au niveau informatique de la mobilité des terminaux est naturellement soulevé par l'étroite relation qui existe entre mobilité et communication. Toutefois, cette prise en compte nécessite au préalable une acquisition.

La nature de cette acquisition varie en fonction du système et des technologies utilisées ainsi que de la richesse des informations requises. Lorsque cette acquisition est accomplie directement par le système, elle n'implique alors pas de recours à une infrastructure ou un système externe. Elle peut être qualifiée d'acquisition $a u$ tonome. Dans le cas contraire, l'utilisation d'un système externe de localisation est requise. L'acquisition est alors dite assistée.

\section{Détection autonome}

La détection autonome se limite souvent à détecter l'impact de la mobilité des terminaux plutôt que la mobilité en elle-même. Elle consiste à évaluer périodiquement la composition du voisinage du terminal considéré. Pour détecter le mouve- 
ment en lui-même, il est nécessaire d'adjoindre aux terminaux les capteurs idoines tels qu'un tachymètre, un accéléromètre ou une boussole. Cela est d'ailleurs proposé dans [39]. Toutefois, par manque de repère fixe commun, l'acquisition de la mobilité ne permet pas de décorreler le mouvement propre du mobile et ceux de ses voisins. Afin d'illustrer les mécanismes de détection autonome, nous envisageons les trois technologies que 802.11 , BlueTooth et le réseau GSM. Nous expliquons les différents mécanismes actifs mis en œuvre pour assurer cette détection.

802.11b: Les réseaux locaux sans-fil [11], ou WLAN, permettent la mise en œuvre de communications sans-fil de moyenne portée. Deux terminaux équipés de cartes $802.11 b$ peuvent communiquer entre eux lorsqu'ils sont au plus éloignés d'environ 250 mètres. Le processus de communication repose sur une technologie à étalement de spectre. Il est de plus symétrique et très semblable à la manière dont ethernet fonctionne : chaque carte dispose d'un identifiant lui permettant de savoir, lors de la réception d'une trame, si elle lui est destinée.

La technologie n'inclut pas de mécanisme actif permettant au système de découvrir quels sont les terminaux voisins. Cela doit être réalisé de manière logicielle par un protocole idoine. Généralement, les solutions proposées reposent sur l'envoi régulier d'un paquet permettant aux terminaux voisins de détecter le terminal émetteur. La fréquence d'émission est fixée selon le type de mobilité envisagée afin de trouver un compromis entre réactivité de la découverte et utilisation de la bande passante disponible.

BlueTooth : BlueTooth [18] est une technologie de communication sans-fil de courte portée. Deux terminaux sont à portée de communication lorsqu'ils sont éloignés d'au plus une dizaine de mètres. Le médium de communication est partagé entre les terminaux par le biais d'un mécanisme en saut de fréquence. Cela en fait une technologie à faible consommation énergétique particulièrement intéressante pour les systèmes embarqués.

La communication entre les terminaux n'est pas symétrique. Elle repose en effet sur la notion de maître et d'esclave. Un terminal maître peut accueillir jusqu'à sept terminaux esclaves au sein de ce que l'on appelle un piconet. Les échanges entre terminaux transitent nécessairement par le terminal maître. Un esclave d'un piconet peut être accessoirement maître d'un autre piconet. Les deux piconets sont alors interconnectés et forment, selon la terminologie BlueTooth, un scatternet. 
Contrairement à 802.11b, BlueTooth dispose d'un mécanisme de découverte afin que les terminaux puissent détecter la présence de leurs pairs. Celui-ci repose sur un mécanisme d'enquête. Pour être détecté par ses voisins, un terminal doit passer dans l'état Inquiry Scan. Toutes les 1, 28 secondes, le terminal tente de recevoir un paquet de découverte durant un centième de seconde sur une fréquence chaque fois différente. Lorsqu'il en reçoit un, il y répond après un délai aléatoire variant de 0 à 1,28 secondes. Pour le détecter, les autres terminaux doivent s'être placés en mode Inquiry et émettre des paquets de découverte.

Ce mécanisme permet aux terminaux de détecter régulièrement leurs voisins et de s'adapter à leur mobilité relative. Du fait de la latence induite par ce mécanisme de découverte, il s'avère assez pénalisant voire inadéquat pour des systèmes s'appuyant sur des terminaux disposant d'une mobilité trop importante.

GSM : Le réseau GSM [24] est un réseau cellulaire de téléphonie mobile particulière développé en Europe. Pour communiquer avec d'autres, chaque utilisateur dispose d'un téléphone portable ou station mobile. Cette station mobile communique avec le réseau par le biais des stations de bases.

Lorsqu'un usager se déplace, il peut être nécessaire qu'il change de station de base parce que celle qu'il utilisait n'offre plus one qualité de transmission suffisante (à cause de l'éloignement, par exemple). Ce processus s'appelle transfert intercellulaire ou handover.

Pour cela, la station mobile effectue un contrôle régulier de la puissance des signaux émis par un ensemble de stations de base dont la liste lui est fournie par celle à laquelle elle est rattachée. C'est par ce contrôle que le terminal acquiert des informations sur sa propre mobilité par rapport aux stations de base environnantes.

Pour décider s'il est nécessaire de demander un transfert au réseau, deux algorithmes sont communément utilisés. Le mobile peut d'une part augmenter sa puissance d'émission, dans les limites autorisées par la norme GSM, jusqu'à ce que cela n'ait plus d'effet. À ce moment là, un tranfert est requis. Cet algorithme est celui de performance minimale. L'algorithme budget de puissance préfère au contraire privilégier la demande de déclenchement du handover à l'augmentation de la puissance.

Les transferts intercellulaires peuvent aussi être déclenchés sur demande du réseau, par exemple dans un but d'équilibrage de charge. 


\section{Acquisition assistée}

L'acquisition assistée de la mobilité des terminaux nécessite l'utilisation d'un service informatique supplémentaire. Elle permet souvent d'effectuer une acquisition plus précise et offre des informations plus riches que celles qui sont délivrées par la détection autonome. Elle peut être classée en deux catégories selon qu'elle est mise en œuvre dans un lieu restreint (acquisition locale) ou bien à l'échelle planétaire (acquisition globale). Ces deux types d'acquisitions peuvent être combinés pour permettre un fonctionnement correct en dépit de leurs limites respectives [26] (par exemple en intérieur $e t$ en extérieur).

Acquisition globale : Le seul système permettant l'acquisition du mouvement de manière globale est actuellement le système Navstar ${ }^{1}$, plus connu sous le nom de $\mathrm{GPS}^{2}$ [12]. Il a pour objectif d'offrir un service de positionnement géographique en n'importe quel point du globe. Un échantillonnage régulier de la position permet d'en déduire des informations sur le mouvement du mobile (vitesse instantanée, cap, etc...)

Le système repose sur la mesure de la distance entre le mobile dont on désire évaluer la position et au moins quatre satellites des vingt-quatre composant la constellation Navstar. Les déplacements des satellites ont été calculés de manière à ce qu'au moins quatre soient visibles depuis le globe terrestre pendant plus de $99 \%$ du temps.

Chaque satellite émet, chaque milliseconde, un message sur deux ondes radioélectriques sur les fréquences de 1,2 et 1,6 GHz. Lorsque le récepteur GPS terrestres reçoit un signal, il calcule la différence de temps entre l'heure d'émission du signal inscrite dans le message et l'heure actuelle. Il calcule alors la distance le séparant du satellite et en déduit une première sphère sur laquelle il se situe. En effectuant ce calcul sur un deuxième satellite sa position se précise puisqu'elle se situe sur un cercle, en l'occurence, l'intersection des deux sphères. Avec trois satellites, seuls deux points subsistent. Le quatrième calcul vient lever l'ambiguïté.

Ce système présente toutefois quelques limites. Il est tout d'abord nécessaire que l'horloge interne du récepteur soit synchronisée avec celles des satellites. Un écart d'une microseconde correspond à une erreur d'environ 300 mètres. D'autre

1. NAVSTAR: NAVigation System with Time And Ranging

2. GPS : Global Positioning System 
part, une ligne de vue directe entre le récepteur est le satellite est nécessaire au fonctionnement. Ce système est donc inopérant en intérieur. Enfin, la vitesse de déplacement des mobiles vient, elle aussi, perturber les mesures de temps de parcours.

Les deux ondes émises par les satellites ont des fréquences différentes. Elles n'évoluent pas de la même façon dans l'atmosphère. Des récepteurs évolués tels que les GPS géodésiques permettent de mesurer la différence de temps de parcours entre les deux ondes et de corriger les aberrations induites par la vitesse du mobile. Les GPS différentiels permettent, quant à eux, de diminuer l'erreur mesurée en utilisant un réseau terrestre de points références dont la position est connue. Dans tous les cas, un fonctionnement à l'intérieur de structures telles que des immeubles est compromis par la nécessité de disposer d'une ligne de vue avec les satellites.

Acquisition locale: L'acquisition locale de la mobilité repose très souvent sur l'acquisition locale de la position. Tout comme avec l'acquisition globale, la mesure régulière de la position permet de déduire des caractéristiques du mouvement telles que la vitesse ou la direction. L'avantage principal de cette approche est qu'elle permet d'obtenir la localisation là où le GPS est inopérant.

Le système Active Bat [46] offre un service local de positionnement. Il permet l'estimation de la distance entre un mobile et des bornes. Cette estimation repose sur l'exploitation de la différence de vitesse de propagation de deux ondes et ressemble à celle réalisée par les récepteurs géodésiques. Les deux ondes utilisées sont les ondes radioélectriques et les ultrasons. La mise en place d'un tel service nécessite toutefois un effort quant au déploiement des bornes et à leur interconnection avec le système centralisé. De plus, cette méthode ne fonctionne qu'en intérieur car l'ultrason est très sensible aux perturbations.

Le système RADAR [3] offre lui aussi un système de localisation intérieure. Il repose sur la mesure de la puissance du signal émis par trois bornes $802.11 b$ et reçu par le mobile. Il nécessite au préalable une phase d'apprentissage conséquent qui consiste en l'échantillonnage de nombreuses positions de la zone couverte. Cela rend son déploiement ardu et nécessite que la zone couverte reste intacte. Par exemple, l'abatage d'une cloison de l'étage couvert d'un immeuble peut avoir des conséquences fatales sur le système.

La réponse au déploiement coûteux est apportée par [6]. Une grille de capteurs formant un système distribué est répartie dans la zone de localisation à couvrir. Chaque capteur est situé à une position connue. La communication entre le mobile 
et les capteurs voisins permet, par le biais d'une modélisation des onde radios, d'estimer la position du mobile. Toutefois, cette méthode n'est valable qu'en extérieur. En effet, la modélisation proposée ne peut tenir compte des phénomènes aléatoires d'écho qui surviennent en intérieur.

\subsection{Systèmes mobiles de communication sans fi I}

Dans la section précédente, nous avons défini la notion à laquelle renvoient les termes de mobilité physique ou encore de mouvement. Nous avons, de plus, détaillé les différentes méthodes de détection informatique de la mobilité des terminaux. Nous présentons à présent les différents types de systèmes de communication sans fil, dans lesquels ces terminaux évoluent traditionnellement. Parmi ces systèmes, on distingue trois principales familles que sont les réseaux cellulaires, les réseaux ad hoc, et enfin les réseaux personnels ${ }^{3}$. Ces systèmes de communication sans fil proposent aux terminaux mobiles qui les composent un schéma particulier de communication. Pour chacun d'eux, nous mettons en avant la relation liant la mobilité physique des terminaux à leur modèle de communication.

\subsubsection{Les réseaux cellulaires}

Les réseaux cellulaires sont des systèmes de communication sans fil qui reposent sur une infrastructure fixe. Les terminaux qui évoluent au sein de ces réseaux doivent obligatoirement s'adresser à cette infrastructure pour pouvoir accéder aux services qu'ils demandent (figure 1). Cet accès s'effectue en général par le biais de bornes qui servent alors de passerelle entre le monde sans fil et le reste du système.

En fonction de la puissance de son émetteur, chaque borne couvre une certaine zone géographique que l'on abstrait en cellule comme illustré dans la figure 2. C'est précisément à cause de cette structuration en cellules que l'on dit que ces systèmes reposent sur des réseaux cellulaires. Généralement, on considère que deux cellules sont voisines lorsqu'il est possible pour un terminal mobile de passer de l'une à l'autre en se déplaçant physiquement, sans passer par une cellule intermédiaire et sans quitter la zone couverte.

L'arrangement spatial des bornes du système définit de facto sa zone de couverture (on suppose en général que l'impact moyen de l'environnement extérieur

3. PAN : Personal Area Networks 


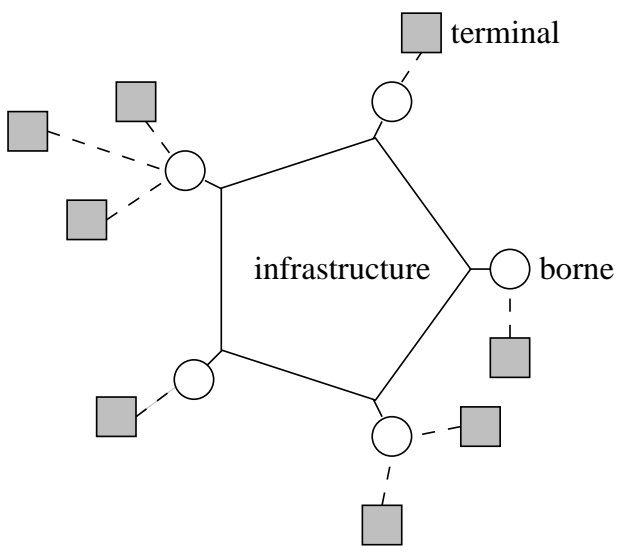

FIG. 1 - Accès par bornes à une infrastructure centralisée

sur les communications sans fil est constant). Cette zone de couverture est souvent considérée comme fixe dans l'espace car les bornes qui la définissent le sont aussi. Il convient donc de répartir judicieusement ces bornes afin que la zone géographique que l'on souhaite couvrir le soit effectivement dans son intégralité.

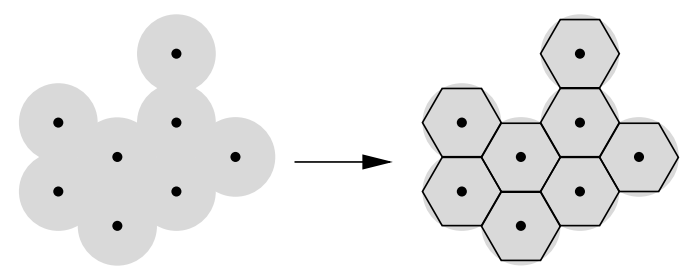

FIG. 2 - Réseau cellulaire

Lorsque le terminal se déplace à l'intérieur de la zone couverte par le réseau cellulaire, il est possible qu'il passe d'une cellule à une autre. Il doit alors changer de borne de référence pour continuer à accéder au réseau. C'est le mécanisme du handover. Pour savoir à quelle borne se rattacher, la solution la plus commune est de choisir celle offrant la meilleure qualité de liaison.

Lorsque la continuité du service offert est requise, ce changement de borne doit s'opérer de la manière la plus transparente possible pour les applications. Des mécanismes doivent donc être mis en œuvre pour permettre la préparation et l'exécution du handover dans les meilleures conditions possibles. Nous reviendrons sur ce pro- 
blème qui illustre une façon dont le mouvement des terminaux peut se manifester dans le système d'informations.

Un des domaines d'application le plus connu des réseaux cellulaires est la téléphonie mobile (et les normes GSM [37] et GPRS [20] en Europe). La norme IEEE $802.11 b$ [11] des réseaux wi-fi permet, elle aussi, de mettre en œvre une telle architecture réseau, avec une portée locale.

\subsubsection{Les réseaux ad hoc}

Un réseau ad hoc est, contrairement aux réseaux cellulaires, un réseau sans infrastructure fixe, formé de terminaux mobiles leur permettant de communiquer les uns avec les autres en dépit de leur éventuelle mobilité. Chaque terminal joue le rôle d'un routeur à part entière et utilise son interface de communication sans fil pour contribuer aux échanges du réseau.

La mobilité des terminaux est a priori non contrainte, tandis que leur portée de communication reste limitée. De ce fait, les mouvements des terminaux ont un impact direct sur la topologie du réseau ad hoc dans lequel ils évoluent. Les routes disponibles entre deux terminaux à un instant donné sont en effet susceptibles d'être modifiées à l'instant suivant, parce qu'un ou plusieurs nœuds intermédiaires ont pu se déplacer et ainsi devenir inaccessibles. Pour permettre à deux terminaux d'échanger des données à travers le réseau de façon suffisamment robuste, il est donc nécessaire de mettre en place des algorithmes de routage capables de détecter les changements topologiques et de s'y adapter. Le mouvement des terminaux est donc un élément important car il influe grandement sur la topologie du réseau.

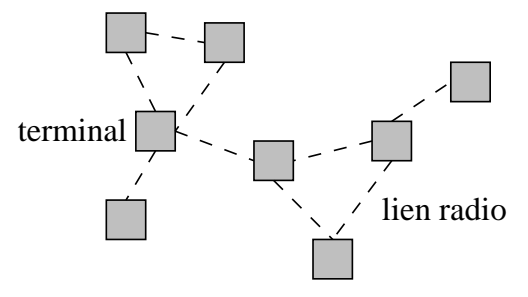

FIG. 3 - Réseau ad hoc

De manière générale, on distingue deux approches principales au problème du routage dans les réseaux ad hoc: l'approche pro-active d'une part, et l'approche 
dite réactive ou encore à la demande d'autre part. De nombreux protocoles ad hoc sont décrits et classés par Feeney [13] selon des critères tels que le modèle de communication ou encore la répartition de l'information de routage. La description exhaustive des divers protocoles ad hoc n'étant pas notre propos, le lecteur s'y référera pour de plus amples informations. Nous allons toutefois rappeler les principes généraux des deux approches principales car nous y ferons référence dans la suite de ce document.

L'approche pro-active: L'objectif des méthodes de routage pro-actives est de maintenir, à travers le réseau, des tables de routage permettant à chaque terminal de connaître à tout moment les routes disponibles dans le réseau de la même manière que cela est réalisé dans les réseaux fixes plus classiques. Contrairement aux réseaux fixes, ces tables de routage sont régulièrement et automatiquement mises à jour, de façon à prendre en compte les modifications topologiques du réseau et de s'y adapter en continu. Pour ce faire, les terminaux échangent régulièrement des messages de contrôle avec leur voisins, c'est-à-dire, les terminaux avec lesquels ils peuvent communiquer directement sans passer par des nœuds relais.

L'avantage de ce type de protocoles est sans conteste le faible délai nécessaire pour obtenir une route entre deux terminaux, ainsi que la rapide connaissance des hôtes inaccessibles. Toutefois, il faut aussi considérer qu'une partie non négligeable des ressources du réseau est réservée à la mise à jour des tables de routage. De plus, une consommation excessive de ressources peut être observée car l'effort de maintien des routes s'effectue que ces dernières soient effectivement utilisées ou non. On notera enfin que le maintien des routes est d'autant plus coûteux que les terminaux sont mobiles car les mises à jour des tables de routage doivent être d'autant plus fréquentes.

Les protocoles pro-actifs les plus classiques sont GSR [8] (Global State Routing), DSDV [34] (Dynamic-Sequenced Distance-Vector) et WRP [31] (Wireless Routing Protocol).

L'approche réactive: Les méthodes réactives (ou à la demande) ne maintiennent pas de manière continue de tables de routage. Une route n'est établie que lorsqu'un nœud en fait expressément la demande. Une première phase de recherche de route (route request) est alors effectuée pour atteindre le terminal destination. S'il est trouvé, le terminal destination répond à l'initiateur de la recherche lors d'une 
seconde phase (route response). Lorsque la source reçoit la réponse, la route est construite et peut être exploitée.

L'avantage des protocoles à la demande est sans conteste la faible surconsommation de ressources réseaux qu'ils entraînent. Ils paraissent donc mieux conçus pour faire face à des changements fréquents de topologie. Toutefois, une fois une route établie doit être maintenue en dépit de la mobilité des routeurs qui la composent, ce qui entraîne là encore un coût en termes de ressources réseaux. D'autre part, il est impossible pour un terminal de savoir a priori quels sont les hôtes qui lui sont inaccessibles. Enfin, le délai d'établissement d'une route est non négligeable et, face à un réseau très dynamique, il peut encore poser des problèmes. En dépit de ces limitations, les protocoles à la demande restent toutefois mieux adaptés que les protocoles pro-actifs aux réseaux dont la topologie varie de manière importante.

Les protocoles à la demande les plus classiques sont DSR [19] (Dynamic Source Routing), AODV [35] (Ad hoc On-demand Distance Vector routing) et TORA [33] (Temporally Ordered Routing Algorithm).

\subsubsection{Les réseaux de personnes}

Les réseaux cellulaires permettent une communication globale sans fil dès que les terminaux qui y participent évoluent au sein d'une zone de couverture. Les réseaux ad hoc offrent la possibilité à des terminaux d'échanger des informations de proche en proche, par le biais de mécanismes de routage, en s'affranchissant d'une zone de couverture prédéfinie, dans la mesure où ils partagent un même voisinage local. Cette notion de voisinage commun à un ensemble de terminaux peut être réduite jusqu'à la considérer uniquement du point de vue d'un terminal. D'une conception macroscopique du monde donnée par les réseaux cellulaires, on glisse ainsi vers la conception microscopique des réseaux de personnes où les seuls nœuds connus a priori sont les terminaux directement voisins (figure 4).

Cette microconnectivité est rendue possible par l'apparition de technologies de communication à moyenne distance telles que $802.11 b$ [11] (environ 250 mètres) puis surtout à courte distance telles que BlueTooth [18] (entre $1 \mathrm{~m}$ et $10 \mathrm{~m}$ ) qui permettent d'envisager l'échange d'informations entre des terminaux mobiles physiquement voisins. Ce type d'échanges se retrouve principalement dans les domaines de l'ubiquité numérique [47], de l'ambient computing [9] ou encore de l'informatique diffuse [4]. Ainsi, les assistants personnels sont destinés à profiter de ce type 


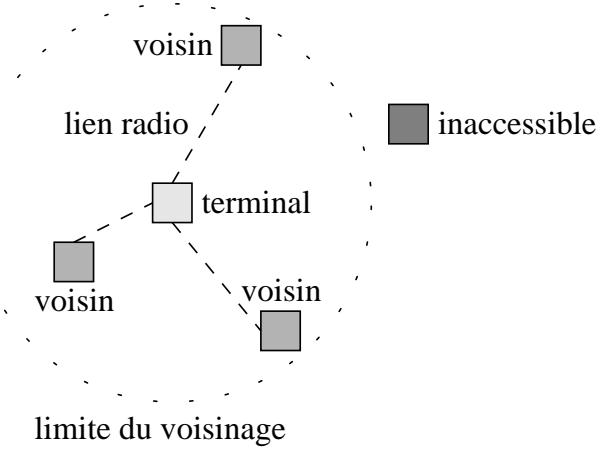

FIG. 4 - Réseau de personnes

de connectivité, afin de permettre à leurs utilisateurs de participer à ce que nous appelons des réseaux de personnes.

Dans un tel contexte, nous verrons que la mobilité des utilisateurs joue, là encore, un rôle important dont il faudra savoir tirer parti.

\subsection{Synthèse}

Après avoir souligné que le terme mobilité renvoie, en informatique, à plusieurs concepts différents selon le domaine dans lequel il est employé, nous avons défini la manière dont nous l'envisageons, à savoir, le mouvement du terminal. Nous avons ensuite explicité la manière dont cette mobilité était captée. Il s'avère que cette acquisition prend deux formes principales que sont la détection autonome et la détection assistée. Nous avons de plus souligné que si la détection assistée demande le recours à un service externe supplémentaire, elle apporte aussi des informations plus précises.

La deuxième partie de ce chapitre a été l'occasion de présenter les grandes classes de systèmes de communication sans fil : les réseaux cellulaires qui disposent d'une infrastructure, et les réseaux qui en sont dépourvus. Parmi ces derniers, on distingue les réseaux ad hoc, dans lesquelles un mécanisme de routage permet la communication entre des terminaux quelconques et les réseaux de personnes qui n'autorisent la communication qu'entre terminaux immédiatement voisins. 
Dans les chapitres suivants, nous allons examiner comment, au sein des ces systèmes, le mouvement des terminaux peut être pris en compte ainsi que l'apport que l'on peut en attendre.

RR $n^{\circ} 4720$ 


\section{Application aux approches adaptatives}

Dans le chapitre précédent, nous avons défini la notion de mobilité physique et donné brièvement les différents moyens techniques permettant sa détection au niveau informatique. Nous avons, de plus, rappelé les principaux types de systèmes de communication sans fil.

Nous entrons à présent au cœur de l'étude proprement dite. Dans cette partie, nous nous attachons à la principale approche au problème de la mobilité dans les systèmes informatiques, à savoir l'approche adaptative. Après en avoir présenté les caractéristiques importantes, nous examinons les différentes manières de prendre en compte la mobilité physique au sein de cette approche.

\subsection{Présentation de l'approche adaptative}

La première grande classe de mécanismes prenant en compte la mobilité physique des terminaux est celle qui regroupe les méthodes dites adaptatives ou palliatives telles qu'elles sont décrites dans [9]. Dans ce genre de systèmes, la mobilité des utilisateurs est perçue comme une source de perturbations entraînant de possibles dégradations du service rendu. L'objectif de tels mécanismes palliatifs est de permettre au système de communication sans fil d'assurer la délivrance d'un service en dépit de cette mobilité. Si l'on prend l'exemple du système de téléphonie mobile, la mobilité d'un usager à travers le territoire est un problème car son terminal n'est pas toujours relié au système par le biais de la même station de base, comme c'est le cas dans un système de téléphonie filaire plus classique. Ce problème est exacerbé lorsque l'usager, pendant qu'il téléphone, se déplace de entrainant aussi un handover. Dans un souci de qualité et de continuité de service, le mécanisme doit être transparent pour l'utilisateur final, et ce, bien qu'un mécanisme de reconfiguration "au vol" soit nécessaire.

Cela nous amène à définir plus précisément les deux types de mobilité que sont mobilité physique et mobilité logique du terminal. La mobilité physique est simple à concevoir, puisqu'elle renvoie directement au mouvement que le terminal effectue dans le monde réel. La mobilité logique, elle, dépend étroitement du système d'informations mis en œuvre et peut être définie comme l'impact de la mobilité physique au niveau du système. D'un point de vue plus géométrique, on peut consi- 
dérer la mobilité logique comme une sorte projection de la mobilité physique par rapport au système d'informations.

Ce parallèle, s'il peut paraître délicat, permet de mieux comprendre pourquoi de tels systèmes adaptatifs ont besoin de prendre en compte la mobilité physique et non pas seulement la mobilité logique, s'ils veulent fonctionner de manière fortement transparente. En effet, il est difficile d'anticiper la mobilité logique, parce que beaucoup d'informations disponibles au niveau de la mobilité physique et utiles à cette anticipation sont oubliées par le système d'informations lors de la projection. Cela est principalement dû au fait qu'elles ne sont pas a priori pertinentes par rapport au service rendu par le système. Si nous reprenons l'exemple du téléphone mobile, les mouvements de l'usager à l'intérieur d'une cellule ne sont pas perçus par le système de téléphonie car ils n'entraînent pas de modification du point de vue du système. Certes, ils entraînent une régulation des puissances de transmission entre téléphone portable et station de base, mais cela ne dépend pas du système téléphonique en lui-même. Ils peuvent pourtant paraître intéressant à étudier si l'on désire anticiper un éventuel handover.

Il faut toutefois tempérer la remarque précédente par le fait qu'il existe des systèmes de prédiction permettant de réagir par anticipation, uniquement en fonction de l'historique de la mobilité logique de l'utilisateur. On trouvera, par exemple, dans [28] et [27], plus d'informations à ce sujet. Cela dépasse toutefois le cadre de ce document qui tente d'explorer les différentes utilisations de la prise en compte de la mobilité physique des utilisateurs. Nous verrons d'ailleurs que cela va au-delà même des mécanismes palliatifs dans le chapitre suivant.

Notons, d'une part, que la mobilité physique n'entraîne pas nécessairement une mobilité logique et que cela dépend du système considéré. D'autre part, la mobilité logique prend une forme différente, selon le type du système de communication sans fil considéré. Ainsi, comme nous l'avons évoqué précédemment, le mouvement physique des terminaux se manifeste par un changement de cellule de référence dans les systèmes cellulaires. Dans les réseaux ad hoc, cette mobilité logique prend l'aspect d'un changement de la configuration des tables de routage. Dans les réseaux de personnes, la mobilité logique correspond, pour un terminal donné, à un changement de l'ensemble de ses voisins. Le tableau 1 résume les différentes façons dont la mobilité physique des terminaux se manifeste suivant les systèmes considérés. 


\begin{tabular}{|l|l|}
\hline Système de communication & Mobilité logique \\
\hline Réseau cellulaire & Changement de cellule de référence \\
Réseau ad hoc & Changement de la topologie du réseau \\
Réseau personnel & Changement de l'ensemble des voisins \\
\hline
\end{tabular}

ТАВ. 1 - Différents types de mobilité logique

L'implantation de mécanismes de prise en compte de la mobilité physique des terminaux mobiles portables se retrouve dans les principaux types de systèmes de communication sans fil, à savoir les réseaux cellulaires, les réseaux ad hoc et les réseaux de personnes. Cette prise en compte du mouvement revêt généralement deux aspects principaux, que sont la prise en compte spatiale et la prise en compte temporelle, que nous allons maintenant décrire.

\subsection{Prise en compte spatiale de la mobilité}

Une première technique de prise en compte du mouvement consiste à détecter la mobilité physique du terminal afin d'en déduire des zones de l'espace possédant de "bonnes" propriétés, au sens de l'application envisagée. Cette approche est utilisée dans les réseaux cellulaires pour permettre de réserver des ressources par anticipation, ainsi que dans les réseaux ad hoc dans le but d'améliorer les performances des protocoles de routage. Nous examinons par la suite la manière dont cette prise en compte spatiale est mise en œuvre au travers de trois exemples.

\subsubsection{Réservation de ressources dans les réseaux cellulaires}

L'un des principaux problèmes qui se posent dans le domaine des réseaux cellulaires est, pour un terminal donné, le transfert intercellulaire ou encore handover. Il survient typiquement lorsqu'un terminal se déplace de telle manière qu'il quitte la cellule dans laquelle il évoluait pour entrer dans une autre. L'accès au réseau doit alors être effectué par la station de base de la nouvelle cellule au détriment de l'ancienne. Lorsque des applications à contraintes temporelles sont en jeu, il est nécessaire que ce processus soit réalisé de façon transparente, c'est-à-dire, sans engendrer de rupture au niveau applicatif. Concrètement, si l'on considère une ap- 
plication telle que la téléphonie, il est important que la communication de l'usager ne soit pas interrompue lorsqu'il change de cellule.

Pour cela, il peut être judicieux de préparer ce changement en réservant les ressources nécessaires dans les cellules susceptibles d'accueillir le terminal. Une méthode simple consiste à effectuer ces réservations dans toutes les cellules voisines. Elle toutefois induit un surcoût très important. En effet, lors du handover, le terminal n'arrivera que dans l'une des cellules voisines. Les autres auront réservé une partie de leurs ressources en pure perte, bloquant éventuellement d'autres entrée de terminaux. Il est en fait possible d'améliorer ce mécanisme de réservation par anticipation en s'appuyant sur le fait qu'en réalité, les terminaux ont rarement un mouvement totalement aléatoire. Ils se déplacent principalement le long de rues ou d'autoroutes. Il suffit alors de ne réserver des ressources que dans la ou les deux cellules dont la probabilité d'accueil du terminal est maximale.

Ce genre de mécanisme a été étudié dans [41]. Un apprentissage préalable est nécessaire, afin que chaque station de base établisse une série de statistiques lui permettant de prédire la future destination d'un terminal en fonction des cellules qu'il vient de traverser. Pour cela, chaque terminal préserve un historique (limité) des cellules qu'il a traversé récemment. Lorsqu'un changement de cellule intervient, la cellule destination en notifie la cellule source afin qu'elle mette à jour ses propre statistiques. Une fois l'apprentissage effectué, les stations de base peuvent limiter la préréservation de ressources aux cellules destination les plus probables.

Les performances d'un tel mécanisme ont été mesurées par simulation dans un système de téléphonie mobile, en considérant la bande passante comme ressource. Les critères suivants ont été mesurés au regard de la charge maximum offerte par chaque cellule: probabilité d'impossibilité du handover, d'impossibilité de traitement d'un nouvel appel, probabilité de rupture de communication et utilisation de la bande passante. La réservation de la ou des deux meilleures cellules donnent des résultats comparables. Elle offre d'autre part, une amélioration raisonnable de la qualité de service sans réservation excessive de bande passante par rapport aux politiques extrêmes de réservation. Elle devrait, selon l'auteur, donner de bien meilleurs résultats lors d'une utilisation réelle. 


\subsubsection{Cas du protocole de routage ad hoc LAR}

Le protocole LAR [23] (pour Location Aided Routing) est un protocole ad hoc de routage à la demande s'appuyant sur la connaissance de paramètres cinématiques du mouvement des nœuds du réseau pour y découvrir des routes. LAR suppose que chaque nœud dispose des moyens de connaître de manière autonome ses coordonnées absolues dans le plan. Il suppose en outre que les nœuds du réseau ont tous une vitesse du même ordre de grandeur dont on connaît la valeur moyenne.

LAR fait partie des protocoles ad hoc dits réactifs, dans lesquels les routes disponibles dans le réseau ne sont pas connues à tout instant, contrairement au cas des protocoles proactifs. Pour connaître les routes, les nœuds n'échangent pas de table de routage de façon régulière. Au contraire, une route n'est établie que lorsqu'un terminal mobile en fait expressément la demande. Un mécanisme de construction de route par inondation du réseau est alors déclenché. L'objectif de LAR est de limiter cette inondation à une zone géographique calculée en fonction des mouvements supposés des deux protagonistes de l'échange, afin de réduire le nombre de messages de contrôle inutiles.

LAR suppose que chaque nœud émet régulièrement ses propres coordonnées et propose d'utiliser pour cela un piggybacking des messages de données émis. Lorsque qu'un nœud source $S$ désire émettre un message $m$ à un nœud destination $D$, LAR définit l'expected zone comme la zone géographique dans laquelle $S$ peut raisonnablement espérer trouver le terminal $D$. À partir de la vitesse moyenne $v$ du terminal et du temps $t$ écoulé depuis la dernière mise à jour de la position de $D$ dans les tables de $S$, l'expected zone est modélisée par le cercle de centre $D$ et de rayon $v t$. Les auteurs évoquent aussi certaines optimisations de cette zone en fonction, par exemple, du cap de $D$.

Une fois cette zone construite, la request zone est calculée de manière à toujours contenir l'expected zone tout entière, ainsi que le nœud source $S$. Cette zone géographique est inscrite dans le message $m$ afin d'être exploitée pour limiter l'inondation du réseau lors de l'envoi de la requête de construction de la route: seuls les nœuds contenus dans cette zone prennent en compte le message de recherche de route et l'émettent à leur tour.

L'appartenance d'un nœud à la request zone est finalement le problème qui va décider du système de routage. Deux politiques différentes sont proposées. La première, illustrée dans la figure 5 consiste à définir la zone comme le plus petit rec- 


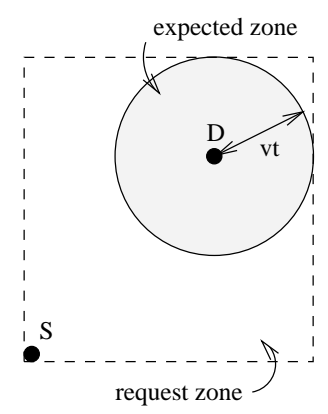

FIG. 5 - LAR: request zone et expected zone

tangle incluant l'expected zone et $S$. La seconde définit l'appartenance d'un nœud à la request zone par le fait que ce dernier doit être plus proche de $D$ que le nœud dont il a reçu le message à convoyer. Cette hypothèse revient en fait à appliquer la première méthode à chaque maillon de la chaîne de recherche comme s'il était le nœud source.

Les performances de ce protocole ont été comparées à celles offertes par un protocole classique fonctionnant par inondation. Pour chacun d'eux, le ratio entre le nombre de paquets relatifs au routage et le nombre de paquets de données proprement dites a été mesuré. Le ratio obtenu par LAR est toujours inférieur au ratio de référence. De plus, il s'accroît moins vite lorsque le nombre de nœuds présents dans le réseau augmente. En outre, l'amélioration de ce ratio tend vers $25 \%$ lorsque la vitesse moyenne des nœuds augmente. Enfin, il se comporte mieux lorsque la connectivité de réseau diminue.

\subsubsection{Cas du protocole de routage ad hoc DREAM}

Contrairement au protocole LAR qui est un protocole ad hoc à la demande, le protocole DREAM [5] (pour Distance Routing Effect Algorithm for Mobility) est, quant à lui, un protocole proactif. Dans ce type de protocoles, les nœuds du réseaux échangent de manière périodique leur table de routage. De fait, les routes disponibles sont connues immédiatement. Ce protocole s'appuie sur la connaissance des mouvements des nœuds du réseau pour enrichir ses tables de routage, afin de diminuer le surcoût réseau qu'il induit dans les communications. Ainsi, en supposant que chaque terminal dispose d'un moyen de connaître sa position et sa vitesse ins- 
tantanée, le protocole propose une méthode de calcul continu des routes, tout en limitant le volume des messages de contrôle nécessaires à leur maintien.

Pour ce faire, les messages de contrôle sont limités à une zone géographique, comme c'est le cas dans le protocole précédent. Toutefois, la méthode de calcul de cette zone diffère quelque peu. Chaque nœud transmet à ses voisins ses caractéristiques cinématiques qui sont datées puis stockées dans la table de routage. Lorsqu'un nœud $A$ doit envoyer un message à un nœud $B$, il consulte sa table de routage et calcule l'angle de sommet $A$ dans lequel $B$ doit se trouver. Il y cherche ensuite ses voisins et leur transmet le message. L'angle de routage (figure 6) est calculé comme suit :

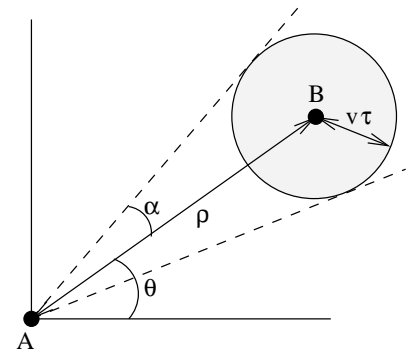

FIG. 6 - DREAM: calcul de l'angle de routage

1. À l'aide des couples $\left(x_{A}, y_{A}\right)$ et $\left(x_{B}, y_{B}\right)$, on calcule aisément $(\rho, \theta)$, les coordonnées polaires de $B$ dans un repère centré en $A$

2. Connaissant le temps écoulé $\tau$ depuis la dernière mise à jour de l'entrée $B$ de la table de routage de $A$ ainsi que la vitesse $v$ de $B$, on calcule le demi-angle au sommet $\alpha$ par $\arcsin (v \tau / \rho)$

Lorsque $\tau$ est supérieur à un certain seuil $\sigma$, l'information contenue dans la table de routage est considérée comme obsolète. On se ramène alors au cas où l'information est absente et une procédure classique de recherche par innondation doit être déclenchée. De même, il n'est pas assuré que $A$ trouve des voisins immédiats dans l'espace angulaire ainsi construit. Lorsque cela se produit, l'algorithme doit recourir à une méthode de recherche de secours par inondation totale du réseau.

Les gains apportés par cette approche ont été mesurés par simulation, pour des réseaux ad hoc offrant une connectivité moyenne de $90 \%$ (c'est-à-dire lorsque 
moins de $10 \%$ des messages émis ne peuvent être acheminés parce qu'aucune route n'existe). Le recours à la procédure d'inondation n'a lieu que dans moins $20 \%$ des cas. Le protocole a, de plus, été comparé au protocole ad hoc DSR. Du point de vue du délai d'acheminement des messages, il apporte un gain de $25 \%$ au minimum et $250 \%$ au maximum.

\subsection{Prise en compte temporelle de la mobilité}

Une seconde technique d'exploitation du mouvement consiste à déduire de la mobilité physique des informations de nature principalement temporelle. Là encore, comme c'est le cas dans les approches palliatives, l'objectif final est d'offrir un service constant, en dépit de la mobilité des terminaux subie par le système.

La prise en compte temporelle du mouvement des terminaux conduit naturellement à l'estimation des durées de voisinages entre eux et le reste du système d'informations. Cette approche est déclinée sous différentes formes dans les réseaux cellulaires (durée de vie du lien entre le terminal et la station de base), les réseaux ad hoc (durée de voisinage entre les routeurs) et les réseaux de personnes (durée de vie du lien entre terminaux). Toutefois, nous verrons qu'elle ne se limite pas à cela, et qu'elle permet le calcul automatique d'autres paramètres temporels. C'est par exemple le cas pour la fréquence d'émission et la durée de vie de messages de contrôle dans les réseaux ad hoc.

\subsubsection{Anticipation du handover dans les réseaux cellulaires}

Le handover, c'est-à-dire le passage d'un terminal d'une cellule à une autre constitue, comme nous l'avons déjà souligné en 3.2.1, un problème difficile, notament au niveau de la réservation de ressources. Une amélioration complémentaire au traitement du handover consiste à prévoir le moment où il va se produire. Or, une station de base ne sait a priori pas quand, pour un terminal donné, le handover va survenir et ne peut donc pas l'anticiper.

Pour cela, un mécanisme d'évaluation du temps minimum restant avant qu'un terminal ne quitte une cellule a été proposé $[2,32]$. Cette technique s'appuie sur la mesure de la puissance du signal lors de la réception par la station de base des paquets réseaux émis régulièrement par le terminal. Mobilité et puissance du signal sont en effet liées. Intuitivement, on conçoit aisément que, plus un émetteur et un 
récepteur sont éloignés, plus le signal reçu sera de faible amplitude. Dans des conditions idéales (vide), cette amplitude est proportionnelle à l'inverse de la distance au carré $\left(1 / d^{2}\right)$ pour de courtes distances (équation de Friss), puis s'écroule en $1 / d^{4}$ pour des distances plus importantes (équation two-ray ground).

L'obtention successive de tels échantillons permet de calculer le taux de variation du signal, c'est-à-dire le rapport entre la variation en amplitude et la variation en temps (i.e. la durée de l'historique). Connaissant ce taux ainsi que la valeur de la puissance du dernier signal reçu, il est simple de calculer le temps restant avant que la puissance du signal ne tombe sous un seuil critique au-dessous duquel on considère que le terminal est en train de quitter la cellule. Le handover doit alors être préparé puis déclenché. Dans un environnement non parfait, il est important d'ignorer les perturbations transitoires qui engendrent des variations à court terme des échantillons. Cela est traditionnellement réalisé avec l'aide d'un filtre passe bas.

La mise en œuvre d'un tel mécanisme permet de diminuer les ruptures lors des handovers en instaurant une priorité de traitement.

\subsubsection{Durée de voisinage dans les réseaux ad hoc}

Le problème du handover est exacerbé dans les réseaux ad hoc puisqu'ils reposent uniquement sur l'interconnexion automatique de routeurs mobiles. La mobilité de ces routeurs est a priori non contrainte. Pourtant, il est parfois raisonnable de considérer qu'elle n'est pas totalement aléatoire et, qu'au contraire, elle suit certains motifs reconnaissables. C'est le cas par exemple, si l'on considère un réseau ad hoc urbain constitué de véhicules automobiles qui ont tendance à se déplacer la plupart du temps en ligne droite le long des rues, et ne bifurquent que de temps à autre. Un autre exemple couramment évoqué pour décrire ce cas de figure est un ensemble de véhicules militaires répartis sur un champ de bataille.

En s'appuyant sur ces motifs non aléatoires du mouvement des terminaux, $\mathrm{Su}$ propose de prédire les durées de voisinages (Link Expiration Time) des nœuds du réseau [41]. Pour cela, chaque terminal transmet régulièrement à ses voisins sa vitesse, son cap et sa position, qu'il obtient par un service de localisation, tel que le GPS, par exemple. Chaque nœud peut ainsi maintenir une table contenant les caractéristiques cinématiques datées de ses voisins. Il devient possible pour les terminaux de prédire le temps d'expiration du lien radio avec chacun de ses voisins, en supposant qu'ils garderont un cap et une vitesse constants à courte échéance. 
Les problèmes relatifs à la synchronisation des horloges sont supposés traités par un protocole idoine, par exemple NTP [30] ou GPS [12].

Considérons un nœud $A$ et son voisin $B$. En supposant que les deux terminaux maintiendront cap et vitesse constants, les équations du mouvement de chaque terminal sont les suivantes:

$$
\begin{aligned}
& \overrightarrow{O A}(t)=\overrightarrow{O A}\left(t_{0}\right)+t \cdot \overrightarrow{V_{A}} \\
& \overrightarrow{O B}(t)=\overrightarrow{O B}\left(t_{0}\right)+t \cdot \overrightarrow{V_{B}}
\end{aligned}
$$

En soustrayant les deux équations, on exprime $\overrightarrow{A B}$ en fonction de $t, \overrightarrow{V_{A}}, \overrightarrow{V_{B}}$ et $t_{0}$. En passant à la norme, on peut isoler $t$. La durée pendant laquelle les deux nœuds resteront voisins correspond au moment où $A$ et $B$ seront au plus distants de $r$, portée de communication radio. Cette durée, exprimée à l'aide des coordonnées, des caps et des vitesses (obtenues via le système GPS et illustré dans la figure 7) est alors donnée par la formule suivante:

$t=\frac{-\left(\Delta v_{x} \cdot \Delta x+\Delta v_{y} \cdot \Delta y\right)+\sqrt{\left(\Delta v_{x}^{2}+\Delta v_{y}^{2}\right) r^{2}-\left(\Delta v_{x} \cdot \Delta y-\Delta v_{y} \cdot \Delta x\right)^{2}}}{\Delta v_{x}^{2}+\Delta v_{y}^{2}}$

avec :

$\Delta x=x_{A}-x_{B}$

$\Delta y=y_{A}-y_{B}$

$\Delta v_{x}=v_{A} \cdot \cos \theta_{A}-v_{B} \cdot \cos \theta_{B}$

$\Delta v_{y}=v_{A} \cdot \sin \theta_{A}-v_{B} \cdot \sin \theta_{B}$

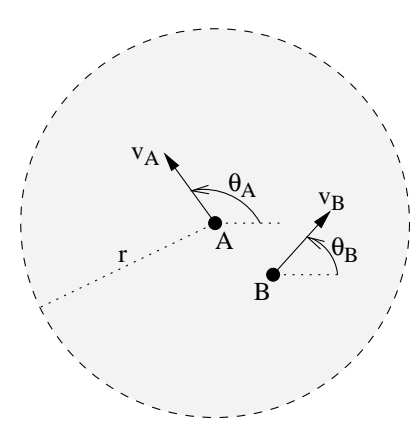

FIG. 7 - calcul du temps d'expiration du lien dans les réseaux ad hoc

RR n ${ }^{\circ} 4720$ 
Su propose d'exploiter cette prédiction de la durée de voisinage afin d'améliorer les protocoles ad hoc proactifs [41], orientés flots [40], ou multicasts [25]. Pour cela, il calcule la durée de vie des routes comme le minimum des durée de vie des sauts qui la compose. Cela permet alors :

1. de choisir les routes les plus stables

2. de prévoir les ruptures imminentes de routes

3. d'établir des routes de remplacement avant les ruptures

Dans ses travaux [41], Su donne les gains apportés par l'utilisation de cette technique. Par rapport à l'utilisation de protocoles ad hoc classiques ne prenant pas en compte la mobilité des routeurs, elle apporte aux réseaux ad hoc orientés flux un meilleur débit. Le délai moyen reste raisonnable, tandis que l'overhead induit est nettement plus faible. Elle offre aux protocoles proactifs (par rapport à DSDV [34], par exemple) un plus haut taux de livraison de paquets. Enfin, en comparaison à d'autres protocoles ad hoc multicasts tels que ODMRP [16], elle permet une diminution du nombre de messages de contrôle nécessaires en cas de faible mobilité et une meilleure utilisation de ces derniers en cas de mobilité soutenue.

\subsubsection{Messages de contrôle dans les réseaux ad hoc}

De manière plus générale, la connaissance pour un terminal de sa propre mobilité permet aussi de calculer automatiquement les paramètres temporels de certaines applications. La fonction de ces paramètres peut varier et il peut s'agir, par exemple, de délais de garde, ou encore, de fréquences d'événements. Pour illustrer cette application de la prise en compte temporelle de la mobilité, nous proposons de revenir sur le protocole de routage DREAM, évoqué en 3.2.3. En effet, en plus de l'amélioration du processus de routage par confinement des messages de contrôle, les auteurs mettent en avant le phénomène de Distance Effect et en proposent une exploitation. Nous expliquons, par la suite, ce qu'est le Distance Effect ainsi que son lien avec la mobilité. Nous montrons enfin son application au calcul automatique de la fréquence d'émission des messages et de leur durée de vie.

Si l'on considère deux terminaux mobiles et que l'on se place du point de vue de l'un d'eux, il semble que l'autre se déplace d'autant plus lentement qu'il est éloigné. D'un point de vue plus géométrique, ce phénomène provient de la propriété angulaire selon laquelle la distance à parcourir pour balayer un angle donné est 
proportionnelle à la distance séparant le mobile du sommet de l'angle. D'autre part, les auteurs observent que plus un nœud du réseau se déplace rapidement, plus il doit fréquemment avertir ses voisins des nouvelles caractéristiques de son mouvement.

Ces observations effectuées, il est possible d'entrevoir certaines améliorations au protocole de routage, du point de vue de la durée de vie des messages de contrôle ainsi que du point de vue de leur fréquence d'émission. Ainsi, les entrées des tables de routage d'un nœud donné peuvent-elles être mises à jour en fonction de la distance le séparant des terminaux correspondants: plus la distance est grande, plus la fréquence de mise à jour peut être réduite, et ceci, sans affecter la validité de la table de routage. Chaque nœud peut, d'autre part, adapter dynamiquement la fréquence d'envoi des messages de contrôle en fonction de sa mobilité, sans toutefois diminuer la précision des routes calculées à partir des tables de routage. En tenant compte conjointement de ces deux propriétés, les auteurs proposent un mécanisme de mise à jour des tables de routage à fréquence variable reposant sur deux types de messages de contrôle que sont les messages à courte durée de vie qui n'atteindront que les voisins les plus proches du nœud émetteur et ceux à longue durée de vie qui, s'ils parcourront la totalité du réseau, seront émis moins souvent.

\subsubsection{Durée de voisinage dans les réseaux de personnes}

À la différence des réseaux ad hoc, les problèmes inhérents à la mobilité physique dans les réseaux de personnes ne se posent pas en terme de routage. Le problème principal posé par le mouvement des terminaux voisins dans les réseaux personnels est que les communications souffrent d'une certaine volatilité. En effet, leur durée de vie varie en fonction des mouvements des terminaux voisins, ainsi que du mouvement propre du terminal considéré.

Afin de limiter l'impact de cette volatilité des liens de voisinage, il peut être envisagé de prendre en compte la mobilité des terminaux. Cela doit permettre d'anticiper les ruptures de communication et ainsi de mieux gérer l'ensemble des échanges ayant lieu au sein du voisinage physique.

Pour ce faire, une architecture logicielle reposant sur l'estimation de la durée de voisinage a été proposée dans le cadre d'interactions de proximité [43]. Compte tenu que les terminaux considérés sont des assistants personnels standards, le recours à un service de localisation externe à été abandonné. Chaque terminal estime donc, de manière autonome, la durée des voisinages auxquels il participe. Pour cela, 


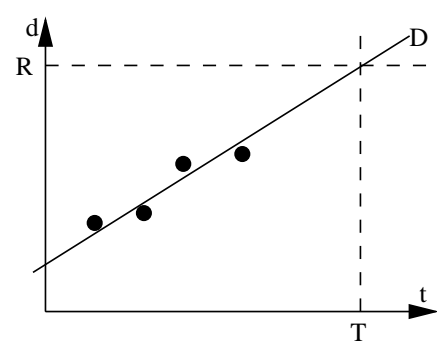

(a) régression linéaire

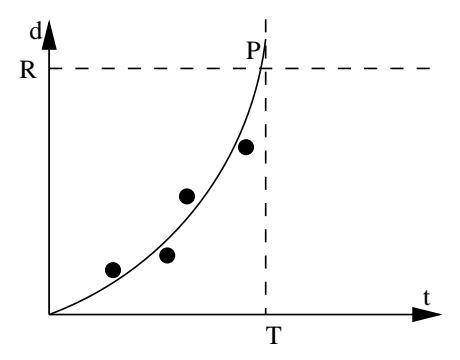

(b) régression non linéaire

FIG. 8 - Prédiction de la durée de voisinage par régression

on suppose qu'un paramètre évoluant en fonction de l'éloignement de deux terminaux est accessible. Ce peut être la puissance des signaux reçus lors des échanges ou bien directement la distance séparant les deux terminaux, si l'on dispose de la technologie idoine. Ce paramètre est ensuite échantillonné et sauvegardé de manière régulière dans un historique de type fenêtre coulissante. Sa taille doit être établie, au départ, en fonction de la mobilité envisagée (mouvements de véhicules, de piétons, etc...).

La prédiction de la durée de voisinage a alors lieu sur l'historique. Un appariement entre l'historique et un ensemble de courbes représentant des mouvements caractéristiques est effectué par régression (linéaire ou non) pour trouver la courbe lui convenant le mieux. Une fois cette courbe $\mathcal{C}$ trouvée et connaissant la portée maximale $R$ de l'interface de communication sans fil du terminal ${ }^{4}$, il devient possible d'estimer la durée $T$ du lien de voisinage en résolvant l'équation $T=\mathcal{C}^{-1}(R)$. Les figures 8(a) et 8(b) illustrent ce mécanisme de prédiction de la durée $T$ en fonction de la distance $R$, de manière linéaire (approche du nuage de mesures par une droite $\mathcal{D}$ lors d'un mouvement uniforme) ou non linéaire (approche du nuage par une parabole $\mathcal{P}$ lors d'une accélération).

Cette technique autonome de prédiction est utilisée par un système d'ordonnancement de messages au sein du voisinage. Elle permet de donner la priorité à des échanges dont on prévoit qu'ils ne seront pas interrompus. Ainsi, pour un même

4. La portée de l'interface de communication sans fil 1 du terminal doit être exprimée selon le paramètre échantillonné. 
volume de données transmises, on constate une économie de la ressource réseau qui peut atteindre jusqu'à 50\% dans les meilleurs cas.

\subsection{Synthèse}

Dans ce chapitre, nous avons étudié les différentes prises en compte de la mobilité physique des terminaux dans les systèmes adaptatifs. Nous avons montré comment cette mobilité physique se manifeste de manière logique au niveau du système d'informations et en quoi l'effort de détection du mouvement peut s'avérer rentable en ce qu'il apporte des informations supplémentaires sur les terminaux.

On distingue dans ces systèmes deux grandes approches de prise en compte du mouvement: la prise en compte spatiale, d'une part, dont l'objectif est de définir des zones géographiques disposant de "bonnes" propriétés pour le système (par exemple, pour le confinement des messages de contrôle) et la prise en compte temporelle, d'autre part, permettant le calcul automatique de paramètres tels que la durée de voisinage ou la fréquence d'émission des messages de contrôle.

Il convient toutefois de souligner que la mobilité est perçue par les systèmes adaptatifs comme une source de perturbations, dont il s'agit d'isoler les applications afin qu'elles fonctionnent convenablement. En effet, les applications mises en œuvre dans ce type de systèmes ne sont pas, a priori, conçues pour intégrer la mobilité des terminaux. Si l'on considère, par exemple, la téléphonie mobile, la fonction principale de l'application est la communication entre les terminaux, quelque soit leur éventuelle mobilité. Dès lors, les différentes prises en compte des mouvements des terminaux constituent toujours un effort supplémentaire à fournir de la part du système.

Ceci n'est toutefois pas obligatoire. Il existe en effet des applications non marginales pour lesquelles cette prise en compte s'effectue sans engendrer de surcoût et de manière naturelle. On trouve la plupart de celles-ci au sein d'un domaine qui a récemment émergé : l'ubiquité numérique. Nous lui consacrons le chapitre suivant. 


\section{Application aux approches ubiquitistes}

Dans les systèmes mobiles de communication sans fil, les approches adaptatives, ou encore palliatives permettent la délivrance d'un ou plusieurs services à des terminaux en dépit de leur mobilité. L'objectif commun de ces approches est la réduction de l'impact de cette mobilité physique au niveau des applications. Ceci est généralement réalisé par une couche système intermédiaire. Nous avons décrit, dans le chapitre précédent, comment la prise en compte du mouvement s'insère au sein de cette couche.

Parallèlement aux approches palliatives existent des approches nouvelles et radicalement différentes, que l'on regroupe à présent sous le nom d'ubiquité numérique, ou encore informatique diffuse. Après en avoir rappelé les principales caractéristiques, nous présentons plusieurs exemples de prise en compte la mobilité physique des terminaux au sein des systèmes ubiquitistes.

\subsection{Présentation de l'approche ubiquitiste}

Les principes généraux de l'ubiquité informatique ont été décrits dans [47]. Son objectif principal est de parvenir à fondre le monde réel, dans lequel les utilisateurs évoluent, et le monde informatique, auquel les terminaux appartiennent, afin de permettre un recours transparent à l'outil informatique. Cette fusion doit s'effectuer de la manière la plus intuitive possible du point de vue des utilisateurs car leur attention et leurs actions sont directement orientées vers le monde physique. C'est donc à l'univers informatique qu'il incombe d'opérer la fusion.

Les premiers projets relatifs à l'ubiquité numérique ont été ParcTab [45], de Rank Xerox, et Active Badge [44], conduit par Olivetti. Active Badge permet la localisation d'utilisateurs munis d'un badge signalant leur présence à une infrastructure de capteurs infrarouges au sein d'un bâtiment. De manière plus générale, ParcTab propose, aux utilisateurs, un accès distribué et transparent au système informatique par le biais d'un terminal et de liaisons infrarouges réparties à l'intérieur d'un bâtiment. Depuis, beaucoup d'études ont été menées sur le sujet. Il existe d'ailleurs de nombreux articles dressant un état de l'art sur le sujet tels que [7] ou [42].

La prise en compte de la mobilité physique, parce qu'elle est fortement corrélée aux actions des utilisateurs, s'inscrit implicitement dans l'ubiquité numérique. De 
nombreuses études, telles que comMotion [29], Cyberguide [1] ou FieldNote [38], exploitent la localisation de l'utilisateur en tant que paramètre à part entière du contexte, c'est-à-dire de l'ensemble des informations caractérisant une situation. Toutefois, comme nous l'avons souligné en 2.1.1, la localisation ne suffit pas à décrire le mouvement physique et l'on constate finalement que peu nombreuses sont les applications ubiquitistes qui exploitent réellement le mouvement de l'utilisateur.

Dans les approches adaptatives, cette prise en compte, si elle s'avère parfois très efficace dans un objectif d'optimisation, n'apparaît que comme une aide supplémentaire au système et, de ce fait, n'entre pas réellement au cœur des applications. Ce raisonnement, poussé à l'extrême, permettrait d'affirmer que, sans cette prise en compte, les applications considérées fonctionneraient toujours, certes, avec des performances moindres.

Les principes de l'ubiquité informatique doivent permettre de dépasser la prise en compte, dans un but exclusivement adaptatif, de la mobilité physique des terminaux. Parce qu'elle tend vers la fusion des univers physique et informatique, l'ubiquité numérique permet la conception d'applications d'un genre nouveau, dans lesquelles le mouvement de l'utilisateur trouve une place fondamentale, quand il devait se contenter d'un rôle secondaire dans les approches palliatives. Nous allons montrer, au travers de deux études, en quoi cette mobilité physique peut trouver sa place dans la sémantique de certaines applications.

\subsection{Adressage dynamique par le mouvement}

De la même façon que des informations sont structurées dans une mémoire informatique, des systèmes d'informations existent implicitement dans le monde réel, structurés par la disposition spatiale des entités. C'est le cas, par exemple, dans les bibliothèques, les supermarchés ou les villes.

S'il l'on dote les entités physiques d'une existence informatique et d'une interface de communication, il devient possible de les adresser dynamiquement: c'est l'utilisateur qui fait l'opération en se déplaçant dans le monde physique.

Cela constitue une conception de la mobilité physique radicalement différente de celle qui est traditionnellement abordée. Au lieu de proposer des solutions visant à s'accomoder de l'impact ${ }^{5}$ de la mobilité physique dans les systèmes d'informations, elle est directement inclue au sein du fonctionnement du système d'infor-

5. Voir la notion de mobilité logique dans le chapitre précédent

RR n ${ }^{\circ} 4720$ 
mations et est envisagée comme un mécanisme d'adressage. Nous décrivons par la suite deux travaux proposant cet adressage dynamique.

\subsubsection{Cooltown}

Cooltown [36] est un projet mené par Hewlett Packard. Son objectif est d'associer une représentation web aux objets de la vie courante. Cela est réalisé par le biais de serveurs HTTP exploitant dans communications sans-fil directes. De ce fait, le mouvement est, dans cette étude, un moyen d'adresser dynamiquement les objets rencontrés dans le monde réel.

Les serveurs HTTP peuvent fonctionner directement sur les entités dont ils assurent la représentation web. Cela est, par exemple, envisageable pour des périphériques tels qu'une imprimante ou un vidéo-projecteur. Dans le cas contraire, le serveur est installé sur un ordinateur déployé spécialement. Une salle de musée peut ainsi proposer une répresentation web des objets d'art qu'elle renferme.

\subsubsection{Spread}

Spread [10] est une étude dont l'objectif est de permettre la gestion informatique d'un système d'informations reposant sur l'espace physique, de la même façon que cela est réalisé pour les processus classiques.

Pour cela, chaque entité physique (utilisateur ou simple objet) prenant part au système rayonne ses informations par le biais d'une interface de communication sans fil au sein d'un volume ${ }^{6}$ modélisé par d'une sphère dont le diamètre dépend de la portée d'émission. Toutes les entités dont la sphère de rayonnement couvre un point de l'espace sont accessibles en ce même point. En se déplaçant, une entité physique perçoit d'autre entités et peut ainsi adresser différentes informations. De ce fait, la mobilité physique d'un utilisateur contribue directement au mécanisme d'adressage du système.

Ce mécanisme d'adressage seul est toutefois insuffisant car plusieurs entités peuvent être disponibles en un même endroit. Il est alors nécessaire de disposer d'un outil supplémentaire pour les différencier. Cela est réalisé par une approche

6. Dans l'absolu, ce volume peut être quelconque, mais la sphère est le volume le plus simple à approcher 
par espace de tuples similaire à LINDA [15]. Cet espace de tuple permet, de plus, de typer les entités.

La figure 9 illustre le concept de système d'informations spatial et montre un consommateur dans un magasin spécialisé. Il est au rayon "films français" et ne voit que trois films. Il peut les différencier en utilisant leur titre (Chacun cherche son chat, Au revoir les enfants et Quelques jours avec moi), fourni par l'espace de tuples. S'il se déplace vers la droite, il atteindra le rayon "films étrangers" et pourra accéder à Blade Runner pour en lire la fiche descriptive, par exemple.

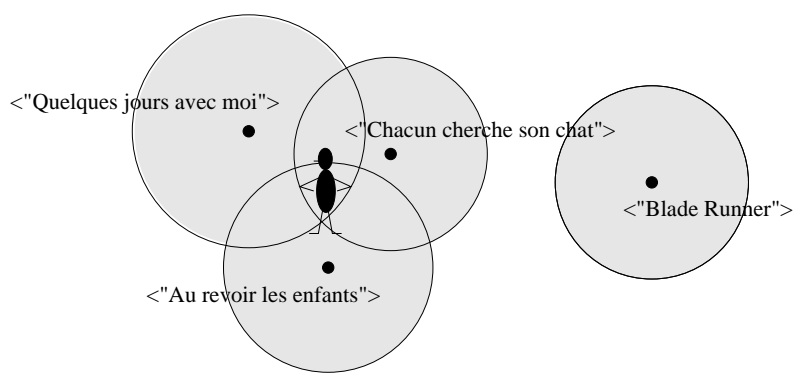

FIG. 9 - Système d'informations spatial

\subsection{Mouvement comme médium de communication}

Dans un article intitulé Pollen: Using people as a communication medium [17], Glance, Snowdon et Meunier présentent un moyen original d'exploiter les déplacements des utilisateurs au sein d'une organisation. L'objectif de l'étude est la création d'un nouveau type de réseau informatique dans lequel les communications ne sont plus exclusivement effectuées par le biais d'une infrastructure filaire, mais peuvent aussi avoir lieu en s'appuyant sur les déplacements des personnes dotées de terminaux mobiles tels que des téléphones ou des assistants personnels. Pour cela, les objets (livres, posters), les périphériques informatiques (imprimantes, fax, vidéoprojecteurs) et les différents lieux de passages (couloirs, restaurant, bureau, salles de réunion) sont équipés d'interfaces de communication sans fil (smart-cards ou $i$-buttons) avec lesquelles les terminaux mobiles des utilisateurs échangent de l'information de manière spontanée et transparente.

Les auteurs comparent ce type de communication à la manière dont les abeilles dispersent le pollen des fleurs en en butinant le nectar. Les utilisateurs arpentent 
durant leur journée différents lieux, consultent divers livres, ou encore utilisent des imprimantes par exemple. Ils chargent ainsi leurs assistants personnels d'informations qui leur sont destinées d'une part, mais aussi d'informations diverses qu'ils transmettent alors à d'autres utilisateurs ou à certains objets, sans avoir à se soucier des échanges. En introduisant l'intelligence et le système de profilage nécessaires, il est de plus possible de contrôler les échanges de messages afin qu'ils atteignent leur cible dans les meilleurs délais. Une mémoire centrale optionnelle, ou ruche, présente sur le réseau câblé classique de l'organisation, peut en outre participer aux échanges lorsque les terminaux sont reliés avec elle (par exemple, lorsque l'assistant personnel est posé sur son socle). Les échanges entre deux nœuds d'un réseau Pollen peuvent ainsi avoir lieu de plusieurs manières, comme illustré sur la figure 10 :

1. directement entre la source et la destination

2. via la ruche

3. via des nœuds intermédiaires
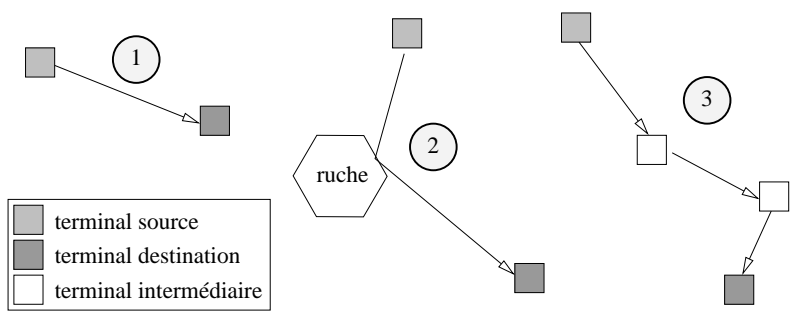

FIG. 10 - Les différents échanges dans un réseau Pollen

Deux classes d'applications semblent pouvoir tirer parti d'un réseau de type Pollen. La première concerne les applications ubiquitistes, c'est-à-dire qui délivrent de l'information contextuelle comme, par exemple, les guides interactifs ou les agendas contextuels. Bien qu'elles ne dépendent pas d'un réseau, elles peuvent en bénéficier. La seconde classe regroupe les applications qui réunissent plusieurs interactions réseau en vue d'accomplir une tâche globale telle que le diagnostic réseau ou la messagerie.

Les principaux intérêts de ce type de réseaux sont leur faible coût en terme d'infrastructure et de périphériques, leur extensibilité et leur configuration facile. Les déplacements des utilisateurs y sont un élément essentiel dans l'acheminement des 
messages et leur mobilité physique est ainsi prise en compte de manière fondamentale.

\subsection{Synthèse}

Au travers de l'étude de deux cas, nous avons remarqué que la prise en compte de la mobilité physique ne se limitait pas uniquement à la résolution d'un problème technique, mais qu'elle pouvait aussi jouer un rôle fondamental dans le fonctionnement de certaines applications.

L'étude Pollen, tout d'abord, propose un nouveau type de réseau informatique. En s'appuyant sur des communications sans fil, il devient possible de faire du mouvement des utilisateurs de moyens informatiques des contributeurs actifs aux échanges du réseau. Leur mobilité devient alors un medium supplémentaire des communications.

Les approches de Spread et Cooltown proposent, quant à elles, un moyen d'adresser des entités ayant une existence informatique. L'espace physique y est envisagé comme un conteneur et la mobilité physique des utilisateurs y devient un moyen d'adressage des informations.

Avec l'ubiquité numérique, la prise en compte de la mobilité pénètre au cœur des applications et en devient un composant essentiel. Pourtant, elle se décline toutefois au travers d'un nombre restreint d'applications. Deux raisons principales expliquent cela. L'informatique diffuse est, d'une part, un domaine relativement récent. Elle n'a pas été aussi profondément étudiée que l'on été les approches adaptatives. D'autre part, la nature même du domaine ralentit la production d'applications prenant en compte le mouvement des terminaux. La conception d'une application originale intégrant la mobilité au niveau sémantique reste, en effet, un exercice difficile. Le domaine demeure cependant très attractif et devrait permettre la conception d'applications offrant des perspectives résolument nouvelles. 


\section{Conclusion}

Dans ce document, nous avons considéré des terminaux informatiques portables, mobiles et dotés d'une interface de communication sans fil. Au travers de plusieurs études de cas, nous avons exploré les différentes techniques de prise en compte leur mouvement au niveau des systèmes d'informations. De cette étude se dégagent deux approches principales, que sont l'approche adaptative ou palliative et l'approche ubiquitiste.

La première catégorie regroupe l'ensemble des systèmes dans lesquels la mobilité physique des terminaux apparaît comme un phénomène dont il s'agit de limiter les répercussions au niveau informatique. La prise en compte de la mobilité est alors une fonctionnalité supplémentaire du système permettant d'améliorer la qualité des services qu'il délivre. Cette prise en compte peut être qualifiée de prise en compte auxiliaire. Elle nécessite toujours un effort logiciel et souvent un effort matériel supplémentaires pour fonctionner. Cette prise en compte, qu'elle repose sur les propriétés spatiales ou temporelles du mouvement, revêt deux aspects principaux que sont la prédiction d'événements en relation avec le mouvement tels que la durée de voisinage et la configuration automatique de paramètres comme la fréquence d'émission de messages de contrôle, par exemple.

La seconde catégorie se retrouve principalement dans le domaine de l'ubiquité numérique. Le mouvement n'est plus considéré comme une source de perturbations du fonctionnement du système, mais, au contraire, en devient un élément essentiel. Sa prise en compte peut être alors considérée comme fondamentale. Les aspects spatiaux et temporels du mouvement ne peuvent être dissociés. C'est une réelle exploitation qui est justifiée de facto par l'existence même du système d'information. Les problèmes soulevés sont généralement d'un autre niveau d'abstraction que ceux exhibés par la prise en compte auxiliaire et la technique est rarement leur aspect principal. Cette approche est résolument nouvelle et se décline, pour le moment, de façon moins prolifique. Ces principaux représentants sont Pollen, où la mobilité est un médium de l'information et Spread et Cooltown, dans lesquels le mouvement est envisagé comme une méthode d'adressage des informations.

La figure 11 dresse un classement des différentes techniques de prise en compte informatique du mouvement selon les critères évoqués précédemment en indiquant, dans les feuilles de l'arbre, les cas étudiés correspondant. 


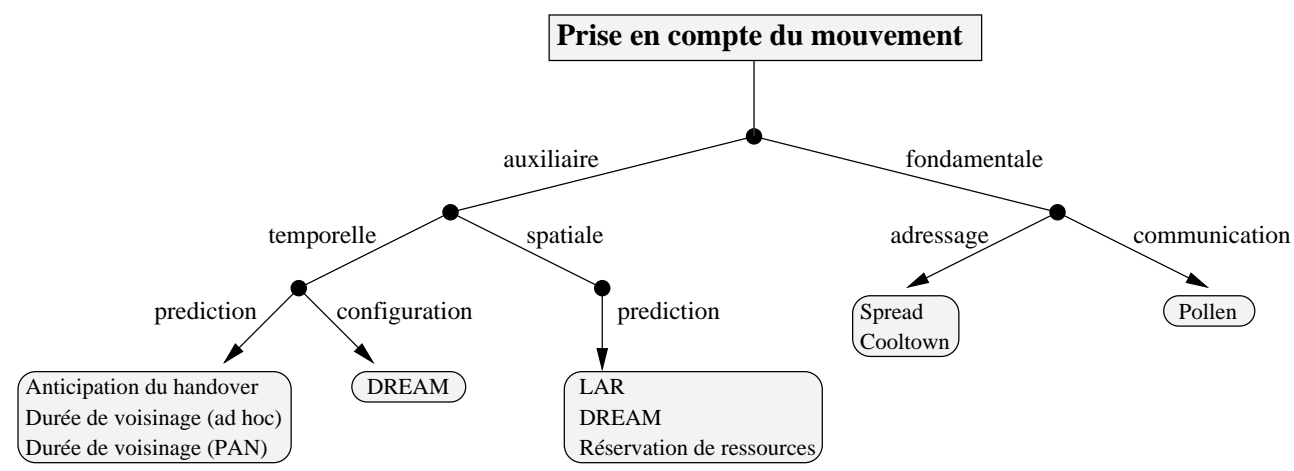

FIG. 11 - Taxonomie de la prise en compte du mouvement

Il est important de noter que si les cas de prise en compte fondamentale restent en nombre limité, ils constituent aussi un véritable renouveau dans le domaine. Ils devraient d'ailleurs permettre la naissance d'applications radicalement novatrices et devraient susciter, de ce fait, un intérêt important. 


\section{Références}

[1] G. D. Abowd, C. G. Atkeson, J. Hong, S. Long, R. Kooper, and M. Pinkerton. Cyberguide: a Mobile Context-Aware Tour Guide. ACM Wireless Networks, 3:421-433, October 1997.

[2] P. Agrawal, D.K. Anvekar, and B. Narendran. Optimal prioritization of handovers in mobile cellular networks. In Proceedings of the fifth international symposium on Personnal Indoor and Mobile Radio Communications (PIMRC'94), volume 4, pages 1393-8. IEEE, September 1994.

[3] Paramvir Bahl and Venkata N. Padmanabhan. Radar: An in-building rf-based user location and tracking system. In Proceedings of the Infocom 2000, volume 2, pages 775-784. IEEE, March 2000.

[4] M. Banâtre and F. Weis. SIS: a new paradigm for mobile computer systems. In Proceedings of the Information Society Technologies Conference (IST'99), November 1999.

[5] Stefano Basagni, Imrich Chlamtac, R. Violet Syrotiuk, and Barry A. Woodward. A Distance Routing Effect Algorithm for Mobility. In Proceedings of the ACM/IEEE International Conference on Mobile Computing and Networking (MOBICOM), pages 49-64, Dallas, TX, USA, October 1998.

[6] N. Bulusu, J. Heidemann, and D. Estrin. Gps-less low cost outdoor localization for very small devices. Personal Communications Magazine, Special Issue on Networking the Physical World, October 2000.

[7] G. Chen and D. Kotz. A Survey of Context-Aware Mobile Computing Research. Technical Report TR2000-381, Department of Computer Science, Dartmouth College, 2000.

[8] Tsu-Wei Chen and Mario Gerla. Global State Routing: A New Routing Scheme for Ad-Hoc Wireless Networks. In Proceedings of the International Conference on Communications (ICC'98), Atlanta, GA, USA, June 1998. IEEE.

[9] P. Couderc, A-M. Kermarrec, and M. Banâtre. Approches adaptatives en mobilité : une synthèse. Technical Report 1276, IRISA, November 1999.

[10] Paul Couderc. Mobilité contextuelle dans les systèmes d'information. PhD thesis, Université de Rennes 1, December 2001. 
[11] B. P. Crow, I. Widjaja, J. G. Kim, and P. T. Sakai. IEEE 802.11 Wireless Local Area Network. IEEE Communications Magazine, 35(9):116-126, September 1997.

[12] P. H. Dana. Global positioning system overview. Available at http://www.colorado.edu/geography/gcraft/notes/gps/gps_f.html, 2000.

[13] Laura Marie Feeney. A Taxonomy for Routing Protocols in Mobile Ad Hoc Networks. Technical Report T99/07, Swedish Institute of Computer Science, Sweden, October 1999.

[14] George H. Forman and John Zahorjan. The challenges of mobile computing. Technical Report TR-93-11-03, Computer Science and Engineering, University of Washington, march 1994.

[15] David Gelernter. Generative communication in linda. ACM Transactions on Programming Languages and Systems, 7(1):80-112, January 1985.

[16] Mario Gerla, G. Pei, Sung-Ju Lee, and C-C Chiang. On-Demand Multicast Routing Protocol (ODMRP) for Ad Hoc Networks. Internet Draft, draft-ietfmanet-odmrp-00.txt, November 1998.

[17] N. Glance, D. Snowdon, and J-L. Meunier. Pollen: Using people as a communication medium. Computer Networks, 35(4):429-442, February 2001.

[18] J. Haartsen, M. Naghshineh, J. Inouye, O. Joeressen, and W. Allen. Bluetooth: Vision, Goals, and Architecture. Mobile Computing and Communications Review, 2(4):38-45, October 1998.

[19] David B. Johnson and David A. Maltz. Dynamic Source Routing in Ad Hoc Wireless Networks. In Imielinski and Korth, editors, Mobile Computing, volume 353. Kluwer Academic Publishers, 1996.

[20] R. Kalden, I. Meirick, and M. Meyer. Wireless Internet Access Based on GPRS. IEEE Personal Communications, 7(2):8-18, April 2000.

[21] Randy H. Katz. Adaptation and mobility in wireless information systems. IEEE Personal Communications, 1:6-17, 1994.

[22] T. Kindberg, J. Barton, J. Morgan, G. Becker, D. Caswell, P. Debaty, G. Gopal, M. Frid, V. Krishnan, H. Morris, J. Schettino, B. Serra, and M. Spasojevic. People, Places, Things: Web Presence for the Real World. In Proceedings of the Third Annual Workshop on Wireless and Mobile Computer Systems and Applications (WMCSA’2000), December 2000. 
[23] Young-Bae Ko and Nitin H. Vaidya. Location-Aided Routing (LAR) in Mobile Ad Hoc Networks. In Proceedings of the ACM/IEEE International Conference on Mobile Computing and Networking (MOBICOM), pages 49-64, Dallas, TX, USA, October 1998.

[24] Xavier Lagrange, Philippe Godlewski, and Sami Tabbane. Réseaux GSMDCS. 4ème edition, 1999.

[25] Sung-Ju Lee, William Su, and Mario Gerla. Ad Hoc Wireless Multicast with Mobility Prediction. In Proceeding of IEEE ICCCN'99, pages 4-9, Boston, MA, USA, October 1999.

[26] Ulf Leonhardt. Supporting Location-Awareness in Open Distributed Systems. $\mathrm{PhD}$ thesis, Department of Computing - Imperial College of Science, Technology and Medicine - University of London, May 1998.

[27] Ben Liang and Zygmunt J. Haas. Prediction Distance-Based Mobility Management for PCS Networks. In Proceedings of INFOCOM'99. IEEE, March 1999.

[28] George Y. Liu and Gerald Q. Maguire Jr. A Class of Mobile Motion Prediction Algorithms for Wireless Mobile Computing and Communications. Mobile Networks and Application, 1(2):113-121, 1996.

[29] N. Marmasse and C. Schmandt. Location-aware information delivery with comMotion. In Proceedings of the Second Symposium on Handheld and Ubiquitous Computing (HUC'2000), pages 157-171, September 2000.

[30] David L. Mills. Internet Time Synchronization: the Network Time Protocol. IEEE Transactions on Communications, 39(10):1482-1493, October 1991.

[31] Shree Murthy and J.J. Garcia-Luna-Aceves. An Efficient Routing Protocol for Wireless Networks. Mobile Networks and Applications, 1(2):183-197, 1996.

[32] B. Narendran, P. Agrawal, and D.K. Anvekar. Minimizing cellular handovers failures without channel utilization loss. In Proceedings of GlobeCOM'94, volume 3, pages 1679-85. IEEE, 1994.

[33] Vincent D. Park and M. Scott Corson. A highly adaptive distributed routing algorithm for mobile wireless networks. In Proceedings of INFOCOM, Kobe, Japan, April 1997. IEEE.

[34] Charles E. Perkins and Pravin Bhagwat. Highly Dynamic DestinationSequenced Distance-Vector Routing (DSDV) for Mobile Computers. In Pro- 
ceeding of the Conference on Communications Architectures, Protocols and Applications (SIGCOMM'94), pages 234-244. ACM, 1994.

[35] Charles E. Perkins and Elizabeth M. Royer. Ad-hoc On-Demand Distance Vector Routing. In Proceedings of the 2nd Workshop on Mobile Computing Systems and Applications, pages 90-100, New Orleans, LA, USA, February 1999. IEEE.

[36] S. Pradhan, C. Brignone, J-H. Cui, A. McReynolds, and M. T. Smith. Websigns: Hyperlinking Physical Locations to the Web. IEEE Computer Magazine, 34(8):42-48, August 2001.

[37] M. Rahnema. Overview Of The GSM System and Protocol Architecture. IEEE Communications Magazine, 31(4):92-100, April 1993.

[38] N. Ryan and J. Pascoe. FieldNote: a Handheld Information System for the Field. In Proceedings of the First International Workshop on TeleGeoProcessing (TeleGeo'99), May 1999.

[39] A. Schmidt and M. Beigl. There is more to context than location: Environment sensing technologies for adaptive mobile user interfaces, 1998.

[40] William Su and Mario Gerla. IPv6 Flow Handoff in Ad Hoc Wireless Networks Using Mobility Prediction. In Proceedings of IEEE GLOBECom'99, pages 271-275, Rio de Janeiro, Brazil, December 1999.

[41] William Wei-Lien Su. Motion Prediction in Mobile/Wireless Networks. PhD thesis, University of California, Los Angeles, CA, USA, 1999.

[42] David Touzet, Frédéric Weis, and Michel Banâtre. Accès à l'information en ubiquité numérique. Technical Report 1460, IRISA, May 2002.

[43] Arnaud Troël, Michel Banâtre, Paul Couderc, and Frédéric Weis. Predictive Scheme for Proximate Interactions. In Proceedings of the International Workshop on Smart Appliances and Wearable Computing (IWSAWC'01), pages 235-239, Mesa, AZ, United States, April 2001.

[44] R. Want, A. Hopper, V. Falcão, and J. Gibbons. The Active Badge Location System. ACM Transactions on Information Systems, 10:91-102, January 1992.

[45] R. Want, B. N. Schilit, N. I. Adams, R. Gold, K. Petersen, D. Goldberg, J. R. Ellis, and M. Weiser. An Overview of the ParcTab Ubiquitous Computing Experiment. IEEE Personal Communications, 2(6):28-33, December 1995. 
[46] A. Ward, A. Jones, and A. Hopper. A new location technique for the active office. In Proceedings of Mobicom'97, September 1997.

[47] M. Weiser. Some Computer Issues in Ubiquitous Computing. Communications of the ACM, 36(7):74-84, July 1993. 
Unité de recherche INRIA Lorraine, Technopôle de Nancy-Brabois, Campus scientifi que, 615 rue du Jardin Botanique, BP 101, 54600 VILLERS LÈS NANCY

Unité de recherche INRIA Rennes, Irisa, Campus universitaire de Beaulieu, 35042 RENNES Cedex

Unité de recherche INRIA Rhône-Alpes, 655, avenue de l'Europe, 38330 MONTBONNOT ST MARTIN

Unité de recherche INRIA Rocquencourt, Domaine de Voluceau, Rocquencourt, BP 105, 78153 LE CHESNAY Cedex

Unité de recherche INRIA Sophia-Antipolis, 2004 route des Lucioles, BP 93, 06902 SOPHIA-ANTIPOLIS Cedex

Éditeur

INRIA, Domaine de Voluceau, Rocquencourt, BP 105, 78153 LE CHESNAY Cedex (France)

http://www.inria.fr

ISSN 0249-6399 